\title{
ON FUZZY TOPOLOGIES GENERATED BY FUZZY RELATIONS
}

\author{
SEEMA MISHRA ${ }^{1}$, REKHA SRIVASTAVA ${ }^{2}, \S$
}

\begin{abstract}
In this paper, we have introduced a fuzzy topology generated by a fuzzy relation as a generalization of the corresponding concept given by Smithson and obtained sufficient conditions under which this fuzzy topology becomes fuzzy $T_{0}$, fuzzy $T_{1}$ and fuzzy $T_{2}$. We have also introduced 'finite intersection property (F.I.P.)' for fuzzy topological spaces and shown that in a fuzzy topological space $(X, \tau)$, this property is equivalent to the Lowen's fuzzy compactness. We have also obtained a sufficient condition under which a fuzzy topology generated by a fuzzy relation becomes fuzzy compact.
\end{abstract}

Keywords: Fuzzy topology, fuzzy relation, separation axioms, fuzzy compactness.

AMS Subject Classification: 54A40.

\section{INTRODUCTION}

Smithson[11] initiated the study of topologies induced by binary relations. Since then many researchers have been working in this area(cf.[6],[4],[1] etc.).

Various types of binary relations have been used to define induced topologies. Dallen and Wattel[3] had obtained a characterization of orderable topologies. Campión, Candeal and Induráin[2] introduced and studied preorderable topologies. Knoblauch[6] introduced topologies induced by a relation $\mathcal{R}$ of a general kind, which are generated by the set of all upper and lower contours of $\mathcal{R}$ taken as a subbase for open sets. He obtained a characterization of a topology on a set $X$ which is induced by a relation of general kind.

Smithson[11] defined a topology on $X$, induced by a relation $\mathcal{R}$, taking the set $\mathcal{S}=$ $D(\mathcal{A}) \cup I(\mathcal{A}) \cup\{X\} \cup\{\phi\}$ as a subbase for closed sets in $X$, where $\mathcal{A}$ is a collection of antisets in $X$, with respect to $\mathcal{R}(A \subseteq X$ is called an antiset if no two distinct elements of $A$ are $\mathcal{R}$-related) and $D(\mathcal{A}), I(\mathcal{A})$ are defined as follows:

$$
D(\mathcal{A})=\{\mathcal{R} A: A \in \mathcal{A}\}, \quad I(\mathcal{A})=\{A \mathcal{R}: A \in \mathcal{A}\}
$$

\footnotetext{
${ }^{1}$ Department of Mathematics, Patna Women's College, Patna University, Patna-800001, India. e-mail: seemamishra.rs.apm12@itbhu.ac.in; ORCID: https://orcid.org/0000-0002-9219-0010.

2 Department of Mathematical Sciences, Indian Institute of Technology, (Banaras Hindu University), Varanasi-221005, India. e-mail: rsrivastava.apm@iitbhu.ac.in; ORCID:https://orcid.org/0000-0002-7694-0352.

$\S$ Manuscript received: September 3, 2019; accepted: January 06, 2020. TWMS Journal of Applied and Engineering Mathematics, Vol.11, No.3 C Işık University, Department of Mathematics, 2021; all rights reserved.
} 
where,

$$
\begin{aligned}
& \mathcal{R} A=\{x:(x, a) \in \mathcal{R}, \text { for some } a \in A\}, \\
& A \mathcal{R}=\{x:(a, x) \in \mathcal{R}, \text { for some } a \in A\} .
\end{aligned}
$$

He obtained sufficient conditions under which the topology induced by a binary relation(in his sense) satisfies separation axioms, compactness and connectedness.

In this paper, we have introduced a fuzzy topology generated by a fuzzy relation, as a generalization of the corresponding concept given by Smithson[11]. In Section 2, we have obtained sufficient conditions under which this generated fuzzy topology will satisfy separation axioms, fuzzy $T_{0}$, fuzzy $T_{1}$ and fuzzy $T_{2}$. In Section 3 , we have introduced 'finite intersection property(F.I.P.)' in a fuzzy topological space and then obtained a characterization of (Lowen's) fuzzy compactness in terms of F.I.P. Using this result, we have obtained a sufficient condition under which a fuzzy topology generated by a fuzzy relation, becomes fuzzy compact.

\section{Preliminaries}

Definition 2.1. [14] A fuzzy set in $X$ is a function $f: X \rightarrow I$, where $I$ is the closed unit interval $[0,1]$. Now we define some basic fuzzy set operations as follows:

Let $f$ and $g$ be two fuzzy sets in $X$. Then

(1) $f=g$ if $f(x)=g(x), \forall x \in X$.

(2) $f \subseteq g$ if $f(x) \leq g(x), \forall x \in X$.

(3) $(f \cup g)(x)=\max \{f(x), g(x)\}, \forall x \in X$.

(4) $(f \cap g)(x)=\min \{f(x), g(x)\}, \forall x \in X$.

(5) $f^{c}(x)=1-f(x), \forall x \in X$ (here $f^{c}$ denotes the complement of $f$ ).

$A$ constant fuzzy set in $X$ taking value $\alpha \in[0,1]$ will be denoted by $\alpha_{X}$.

Definition 2.2. [9] Let $\Omega$ be an index set and $\left\{f_{i}: i \in \Omega\right\}$ be a family of fuzzy sets in $X$. Then their union $\bigcup_{i \in \Omega} f_{i}$ and intersection $\bigcap_{i \in \Omega} f_{i}$ are defined respectively as follows:

(1) $\left(\bigcup_{i \in \Omega} f_{i}\right)(x)=\sup \left\{f_{i}(x): i \in \Omega\right\}, \forall x \in X$.

(2) $\left(\bigcap_{i \in \Omega} f_{i}\right)(x)=\inf \left\{f_{i}(x): i \in \Omega\right\}, \forall x \in X$.

Definition 2.3. [12] A fuzzy point $x_{\lambda}(0<\lambda<1)$ in $X$ is a fuzzy set in $X$ such that

$$
x_{\lambda}\left(x^{\prime}\right)= \begin{cases}\lambda, & \text { if } x^{\prime}=x \\ 0, & \text { otherwise. }\end{cases}
$$

Here $x$ and $\lambda$ are respectively called the support and value of $x_{\lambda}$. A fuzzy point $x_{\lambda}$ is said to belong to a fuzzy set $f$ if $\lambda<f(x)$ and two fuzzy points $x_{r}$ and $y_{s}$ in $X$ are said to be distinct if $x \neq y$.

Definition 2.4. [7] A fuzzy topological space is a pair $(X, \tau)$ consisting of a non empty set $X$ and a family $\tau$ of fuzzy sets in $X$ satisfying the following conditions:

(1) $\alpha_{X} \in \tau, \forall \alpha \in[0,1]$;

(2) If $\left\{f_{i}: i \in \Omega\right\}$ is an arbitrary family of fuzzy sets in $\tau$, then $\bigcup_{i \in \Omega} f_{i} \in \tau$.

(3) If $f, g \in \tau$, then $f \cap g \in \tau$.

Then $\tau$ is called a fuzzy topology on $X$ and the members of $\tau$ are called fuzzy open sets (or $\tau-$ fuzzy open sets). A fuzzy set $f$ in $X$ is called fuzzy closed if $f^{c} \in \tau$. 
Definition 2.5. [14] A fuzzy relation $\mathcal{R}$ on $X$ is a fuzzy set in $X \times X$ i.e., $\mathcal{R}$ is a mapping from $X \times X$ to $[0,1]$.

Two elements $x, y$ of $X$ are said to be $\mathcal{R}$-related if $\mathcal{R}(x, y)>0$.

Definition 2.6. [15] A fuzzy relation $\mathcal{R}$ on $X$ is said to be

(1) reflexive if $\mathcal{R}(x, x)=1$, for each $x \in X$;

(2) antisymmetric if $\mathcal{R}(x, y)>0$ and $\mathcal{R}(y, x)>0$ implies that $x=y$;

(3) transitive if $\mathcal{R}(x, z) \geq \min \{\mathcal{R}(x, y), \mathcal{R}(y, z)\}$, for each $x, y, z \in X$;

Definition 2.7. [15] A fuzzy relation in $X$ is said to be a fuzzy partial order if it is reflexive, antisymmetric and transitive.

Definition 2.8. Let $X$ be a non empty set and $A \subseteq X$. Then $A$ is called an $\mathcal{R}$-antiset of $X$ if no two distinct elements of $A$ are $\mathcal{R}$-related.

Definition 2.9. Let $X$ be a non empty set, $\mathcal{R}$ be a fuzzy relation on $X$ and $A \subseteq X$. Then fuzzy sets $L_{A}$ and $R_{A}$ on $X$, are defined as follows:

$$
\begin{aligned}
& L_{A}(y)=\sup _{a \in A} \mathcal{R}(y, a), \\
& R_{A}(y)=\sup _{a \in A} \mathcal{R}(a, y),
\end{aligned}
$$

for each $y \in X$.

Smithson[11] introduced and studied a topology on a set $X$, induced by a relation $\mathcal{R}$. Here we generalize this concept in the fuzzy setting.

Definition 2.10. Let $\mathcal{R}$ be a fuzzy relation on $X$ and $\mathcal{A}$ be a collection of $\mathcal{R}$-antisets of $X$. Then $\tau_{\mathcal{R}, \mathcal{A}}$ is the fuzzy topology on $X$ generated by taking

$$
\mathcal{S}=\left\{L_{A}\right\}_{A \in \mathcal{A}} \cup\left\{R_{A}\right\}_{A \in \mathcal{A}} \cup\left\{\alpha_{X}: \alpha \in[0,1]\right\},
$$

as a subbase for fuzzy closed sets in $X$ i.e., every fuzzy closed set in $X$ can be written as an intersection of finite unions of members of $\mathcal{S}$.

Example 2.1. Let $\mathcal{R}$ be a fuzzy relation on $X=\{a, b\}$, which is given as follows:

\begin{tabular}{|l|c|r|}
\hline $\mathcal{R}$ & $a$ & $b$ \\
\hline$a$ & 0.1 & 0 \\
\hline$b$ & 0 & 0.3 \\
\hline
\end{tabular}

and $\mathcal{A}=\{\{a\},\{a, b\}\}$. Then the fuzzy topology $\tau_{\mathcal{R}, \mathcal{A}}$ is generated by the following subbase $\mathcal{S}$ for the fuzzy closed sets in $X$ :

$$
\mathcal{S}=\left\{L_{\{a\}}, L_{\{a, b\}}, R_{\{a\}}, R_{\{a, b\}}\right\} \cup\left\{\alpha_{X}: \alpha \in[0,1]\right\},
$$

where $L_{\{a\}}, L_{\{a, b\}}, R_{\{a\}}, R_{\{a, b\}}$ are given by:

$$
L_{\{a\}}=\frac{0.1}{a}+\frac{0}{b}, \quad L_{\{a, b\}}=\frac{0.1}{a}+\frac{0.3}{b}, \quad R_{\{a\}}=\frac{0.1}{a}+\frac{0}{b}, \quad R_{\{a, b\}}=\frac{0.1}{a}+\frac{0.3}{b} .
$$

So, the collection $\mathcal{H}$ of fuzzy closed sets in $X$ consists of arbitrary intersections of finite unions of members of $\mathcal{S}$ and hence $\tau_{\mathcal{R}, \mathcal{A}}$ is the set obtained by taking complements of members of $\mathcal{H}$.

We mention here that in [10], for a given fuzzy relation $\mathcal{R}$ on $X$, the authors have introduced and studied the fuzzy topology $\tau$ on $X$ generated by $\left\{L_{\{x\}}, R_{\{x\}}\right\}_{x \in X}$ considered as a subbase for fuzzy open sets in $X$, i.e, every member of $\tau$ is a union of finite intersections of members of the set $\left\{L_{\{x\}}, R_{\{x\}}\right\}_{x \in X}$. This is a generalization of the corresponding concept given in $[6]$. 
Definition 2.11. Let $(X, \tau)$ be a fuzzy topological space. Then $(X, \tau)$ is said to be

(1) fuzzy $T_{0}$ if for each $x, y \in X$ such that $x \neq y$, there exists a fuzzy closed set $U$ in $X$ such that $U(x) \neq U(y)$;

(2) fuzzy $T_{1}$ if for each $x, y \in X$ such that $x \neq y$, there exist two fuzzy closed sets $U, V$ such that $U(x)=1, U(y)=0, V(x)=0, V(y)=1$;

(3) fuzzy $T_{2}$ if for each pair of distinct fuzzy points $x_{r}, y_{s}$ in $X$, there exist two fuzzy closed sets $U, V$ such that $r>U(x), s>V(y)$ and $U \cup V=1_{X}$.

We remark here that (1), (2), (3) given above are equivalent to the definitions of fuzzy $T_{0}$, fuzzy $T_{1}$ and fuzzy $T_{2}$ given in [8], [13], [12], respectively.

Definition 2.12. [7] Let $(X, \tau)$ be a fuzzy topological space. Then a fuzzy set $f$ in $X$ is said to be fuzzy compact if for any $\beta \subseteq \tau$ such that $\bigcup_{\mu \in \beta} \mu \supseteq f$ and for $\epsilon>0$, there exists a finite subfamily $\beta_{0} \subseteq \beta$ such that $\bigcup_{\mu \in \beta_{0}} \mu \supseteq f-\epsilon$.

A fuzzy topological space $(X, \tau)$ is said to be fuzzy compact if each constant fuzzy set in $X$ is fuzzy compact.

Proposition 2.1. [13] Let $(X, \tau)$ be a fuzzy topological space. Then the following statements are equivalent:

(1) $(X, \tau)$ is fuzzy $T_{1}$.

(2) $\{x\}$ is fuzzy closed, $\forall x \in X$.

\section{Separation AXioms}

In this section, we prove some results in the fuzzy setting, which are counterparts of the corresponding results given in [11]. In our discussion here, we shall assume that $\mathcal{R}$ is a fuzzy relation on $X$ and $\mathcal{A}$ is a collection of $\mathcal{R}$-antisets.

Proposition 3.1. Let for each $x, y \in X$ such that $x \neq y$, there exist $z \in X$ such that $\mathcal{R}(x, z) \neq \mathcal{R}(y, z) \quad$ or $\mathcal{R}(z, x) \neq \mathcal{R}(z, y))$ and $\mathcal{A}$ contains singletons. Then $\left(X, \tau_{\mathcal{R}, \mathcal{A}}\right)$ is fuzzy $T_{0}$.

Proof. Let $x, y \in X$ such that $x \neq y$. Then by our assumption, there exists $z \in X$ such that $\mathcal{R}(x, z) \neq \mathcal{R}(y, z)$ which implies that $L_{\{z\}}(x) \neq L_{\{z\}}(y)$. Since $L_{\{z\}}$ is fuzzy closed in $\tau_{\mathcal{R}, \mathcal{A}}$ and is such that $L_{\{z\}}(x) \neq L_{\{z\}}(y)$, so $\left(X, \tau_{\mathcal{R}, \mathcal{A}}\right)$ is fuzzy $T_{0}$.

Similarly, we can proceed for the case when $\mathcal{R}(z, x) \neq \mathcal{R}(z, y)$.

Example 3.1. Let $\mathcal{R}$ be a fuzzy relation on $X=\{a, b\}$, which is given as follows:

\begin{tabular}{|l|c|c|}
\hline $\mathcal{R}$ & $a$ & $b$ \\
\hline$a$ & 0.7 & 0.3 \\
\hline$b$ & 0.6 & 0.5 \\
\hline
\end{tabular}

and $\mathcal{A}=\{\{a\},\{b\}\}$. Then the fuzzy topology $\tau_{\mathcal{R}, \mathcal{A}}$ is generated by the following subbase $\mathcal{S}$ for the fuzzy closed sets in $X$ :

$$
\mathcal{S}=\left\{L_{\{a\}}, L_{\{b\}}, R_{\{a\}}, R_{\{b\}}\right\} \cup\left\{\alpha_{X}: \alpha \in[0,1]\right\},
$$

where $L_{\{a\}}, L_{\{b\}}, R_{\{a\}}, R_{\{b\}}$ are given by:

$$
L_{\{a\}}=\frac{0.7}{a}+\frac{0.6}{b}, \quad L_{\{b\}}=\frac{0.3}{a}+\frac{0.5}{b}, \quad R_{\{a\}}=\frac{0.7}{a}+\frac{0.3}{b}, \quad R_{\{b\}}=\frac{0.6}{a}+\frac{0.5}{b} .
$$

Note that $\left(X, \tau_{\mathcal{R}, \mathcal{A}}\right)$ is fuzzy $T_{0}$ since for $a, b \in X, a \neq b$, there exists a fuzzy closed set $U=L_{\{a\}}$ in $X$ such that $U(a) \neq U(b)$. 
Proposition 3.2. Let for each $x \in X$ and $y \in X \backslash\{x\}$, there exists $z \in X$ such that $\mathcal{R}(x, z)=1($ or $\mathcal{R}(z, x)=1)$ and $\mathcal{R}(y, z)=0($ or $\mathcal{R}(z, y)=0)$. Then $\mathcal{A}$ contains singletons implies that $\left(X, \tau_{\mathcal{R}, \mathcal{A}}\right)$ is fuzzy $T_{1}$.

Proof. In view of Proposition 2.1, we show that $\{x\}$ is fuzzy closed. Let $y_{r} \in X \backslash\{x\}$. Then $x \neq y$. So according to our assumption, there exists $z \in X$ such that $\mathcal{R}(x, z)=1$ and $\mathcal{R}(y, z)=0$, which implies that $L_{\{z\}}(x)=1$ and $L_{\{z\}}(y)=0$. Therefore, there exists $L_{\{z\}}^{c} \in \tau_{\mathcal{R}, \mathcal{A}}$ such that $y_{r} \in L_{\{z\}}^{c} \subseteq X \backslash\{x\}$, which implies that $\{x\}$ is fuzzy closed.

The other case can be handled similarly.

Corollary 3.1. Let $\mathcal{R}$ be a fuzzy relation on $X$ which is reflexive and antisymmetric. Then $\mathcal{A}$ contains singletons implies that $\left(X, \tau_{\mathcal{R}, \mathcal{A}}\right)$ is fuzzy $T_{1}$.

Proof. Let $x, y \in X$ such that $x \neq y$. Then by the antisymmetry of $\mathcal{R}$, either $\mathcal{R}(x, y)=0$ or $\mathcal{R}(y, x)=0$. Also by the reflexivity of $\mathcal{R}, \mathcal{R}(x, x)=1$, for each $x \in X$. In both the cases, if we set $z=x$, then by Proposition $3.2,\left(X, \tau_{\mathcal{R}, \mathcal{A}}\right)$ is fuzzy $T_{1}$.

Definition 3.1. A collection $\mathcal{A}$ of $\mathcal{R}$-antisets is called separating if for each $x \in X$ and $y \in X \backslash\{x\}$, there exists $A \in \mathcal{A}$ such that $L_{A}(x)=1$ and $L_{A}(y)=0$ or $R_{A}(x)=1$ and $R_{A}(y)=0$.

Proposition 3.3. If $\mathcal{A}$ is separating, then $\left(X, \tau_{\mathcal{R}, \mathcal{A}}\right)$ is fuzzy $T_{1}$.

Proof. Let $x, y \in X$ such that $x \neq y$. Then $y \in X \backslash\{x\}$ and so by our assumption, there exists $A \in \mathcal{A}$ such that $L_{A}(x)=1$ and $L_{A}(y)=0$ or $R_{A}(x)=1$ and $R_{A}(y)=0$. Similarly, for $x \in X \backslash\{y\}$, there exists $A^{\prime} \in \mathcal{A}$ such that $L_{A^{\prime}}(y)=1$ and $L_{A^{\prime}}(x)=0$ or $R_{A^{\prime}}(y)=1$ and $R_{A^{\prime}}(x)=0$. Since $L_{A}, L_{A^{\prime}}, R_{A}, R_{A^{\prime}}$ are fuzzy closed sets in $X$, therefore $\left(X, \tau_{\mathcal{R}, \mathcal{A}}\right)$ is fuzzy $T_{1}$.

Example 3.2. Let $\mathcal{R}$ be a fuzzy relation on $X=\{a, b\}$, which is given as follows:

\begin{tabular}{|c|c|c|c|}
\hline $\mathcal{R}$ & $a$ & $b$ & $c$ \\
\hline$a$ & 1 & 0.3 & 0.4 \\
\hline$b$ & 0 & 1 & 0 \\
\hline$c$ & 0 & 0.5 & 1 \\
\hline
\end{tabular}

and $\mathcal{A}=\{\{a\},\{b\},\{c\}\}$. Then the fuzzy topology $\tau_{\mathcal{R}, \mathcal{A}}$ is generated by the following subbase $\mathcal{S}$ for the fuzzy closed sets in $X$ :

$$
\mathcal{S}=\left\{L_{\{a\}}, L_{\{b\}}, L_{\{c\}}, R_{\{a\}}, R_{\{b\}}, R_{\{c\}}\right\} \cup\left\{\alpha_{X}: \alpha \in[0,1]\right\},
$$

where $L_{\{a\}}, L_{\{b\}}, L_{\{c\}}, R_{\{a\}}, R_{\{b\}}, R_{\{c\}}$ are given by:

$$
\begin{aligned}
& L_{\{a\}}=\frac{1}{a}+\frac{0}{b}+\frac{0}{c}, \quad L_{\{b\}}=\frac{0.3}{a}+\frac{1}{b}+\frac{0.5}{c}, \quad L_{\{c\}}=\frac{0.4}{a}+\frac{0}{b}+\frac{1}{c}, \\
& R_{\{a\}}=\frac{1}{a}+\frac{0.3}{b}+\frac{0.4}{c}, \quad R_{\{b\}}=\frac{0}{a}+\frac{1}{b}+\frac{0}{c},
\end{aligned}
$$

Since, $L_{\{a\}}=\{a\}, R_{\{b\}}=\{b\}$ and $L_{\{c\}} \cap R_{\{c\}}=\{c\}$ are fuzzy closed sets in $X$, so in view of the Proposition 2.1, $\left(X, \tau_{\mathcal{R}, \mathcal{A}}\right)$ is fuzzy $T_{1}$.

Definition 3.2. A collection $\mathcal{A}$ of $\mathcal{R}$-antisets completely separates points of $X$ if for each $x, y \in X$ such that $x \neq \neq_{k} y$, there exist $B_{1}, B_{2}, \ldots, B_{k}$, where for each $i, B_{i}=L_{A}$ or $R_{A}$, $A \in \mathcal{A}$ such that $1_{X}=\bigcup_{i=1}^{k} B_{i}$ and $B_{i}(x)>0$ implies that $B_{i}(y)=0$.

Theorem 3.1. If $\mathcal{A}$ completely separates points of $X$, then $\left(X, \tau_{\mathcal{R}, \mathcal{A}}\right)$ is fuzzy $T_{2}$. 
Proof. Let $x_{r}$ and $y_{s}$ be two distinct fuzzy points in $X$. Then $x \neq y$. Since $\mathcal{A}$ completely separates points of $X$, so there exist $B_{1}, B_{2}, \ldots, B_{k}$, where for each $i, B_{i}=L_{A}$ or $R_{A}$, $A \in \mathcal{A}$ such that

$$
1_{X}=\bigcup_{i=1}^{k} B_{i}
$$

and $B_{i}(x)>0$ implies that $B_{i}(y)=0$. Since $1_{X}=\bigcup_{i=1}^{k} B_{i}$, so for $x, y \in X$, there exist $i_{1}$ and $i_{2}$ such that $B_{i_{1}}(x)=1$ and $B_{i_{2}}(y)=1$. Next, since $B_{i_{1}}(x)=1>0$, so $B_{i_{1}}(y)=0$. Let $X_{1}=\cup\left\{B_{i} \mid B_{i}(y)=0\right\}$ and $X_{2}=\cup\left\{B_{i} \mid B_{i}(y)>0\right\}$. Here $X_{1}(y)=0, X_{2}(x)=0$ and in view of (1), we have $X_{1} \cup X_{2}=1_{X}$. Note that $X_{1}$ and $X_{2}$ are fuzzy closed sets in $X$ such that $r>X_{2}(x), s>X_{1}(y)$ and $X_{1} \cup X_{2}=1_{X}$. Therefore, $\left(X, \tau_{\mathcal{R}, \mathcal{A}}\right)$ is fuzzy $T_{2}$.

Example 3.3. Let $\mathcal{R}$ be a fuzzy relation on $X=\{a, b, c\}$, which is given as follows:

\begin{tabular}{|c|c|c|c|}
\hline $\mathcal{R}$ & $a$ & $b$ & $c$ \\
\hline$a$ & 1 & 0 & 0.3 \\
\hline$b$ & 0.7 & 1 & 0 \\
\hline$c$ & 0 & 0.8 & 1 \\
\hline
\end{tabular}

and $\mathcal{A}=\{\{a\},\{b\},\{c\}\}$. Then the fuzzy topology $\tau_{\mathcal{R}, \mathcal{A}}$ is generated by the following subbase $\mathcal{S}$ for the fuzzy closed sets in $X$ :

$$
\mathcal{S}=\left\{L_{\{a\}}, L_{\{b\}}, L_{\{c\}}, R_{\{a\}}, R_{\{b\}}, R_{\{c\}}\right\} \cup\left\{\alpha_{X}: \alpha \in[0,1]\right\},
$$

where $L_{\{a\}}, L_{\{b\}}, L_{\{c\}}, R_{\{a\}}, R_{\{b\}}, R_{\{c\}}$ are given by:

$$
\begin{aligned}
& L_{\{a\}}=\frac{1}{a}+\frac{0.7}{b}+\frac{0}{c}, \quad L_{\{b\}}=\frac{0}{a}+\frac{1}{b}+\frac{0.8}{c}, \quad L_{\{c\}}=\frac{0.3}{a}+\frac{0}{b}+\frac{1}{c}, \\
& R_{\{a\}}=\frac{1}{a}+\frac{0}{b}+\frac{0.3}{c}, \quad R_{\{b\}}=\frac{0.7}{a}+\frac{1}{b}+\frac{0}{c}, \quad R_{\{c\}}=\frac{0}{a}+\frac{0.8}{b}+\frac{1}{c} .
\end{aligned}
$$

Since for the fuzzy points $x_{r}, y_{s} \in X$, there exist two fuzzy closed sets $U=L_{\{b\}} \cup R_{\{c\}}$ and $V=L_{\{c\}} \cup R_{\{a\}}$ in $X$ such that $r>U(x), s>V(y)$ and $U \cup V=1_{X}$, for the fuzzy points $y_{r}, z_{s} \in X$, there exist two fuzzy closed sets $U=L_{\{c\}} \cup R_{\{a\}}$ and $V=L_{\{a\}} \cup R_{\{b\}}$ in $X$ such that $r>U(y), s>V(z)$ and $U \cup V=1_{X}$ and for the fuzzy points $z_{r}, x_{s} \in X$, there exist two fuzzy closed sets $U=L_{\{a\}} \cup R_{\{b\}}$ and $V=L_{\{b\}} \cup R_{\{c\}}$ in $X$ such that $r>U(z)$, $s>V(x)$ and $U \cup V=1_{X}$, so $\left(X, \tau_{\mathcal{R}, \mathcal{A}}\right)$ is fuzzy $T_{2}$.

\section{Finite intersection PROPERTy AND FUZZy COMPACtNesS}

In this section, we introduce 'finite intersection property' in fuzzy topological spaces and find a sufficient condition under which a fuzzy topology generated by a fuzzy relation, becomes fuzzy compact.

Definition 4.1. A family $\mathcal{F}$ of fuzzy sets is said to satisfy the finite intersection property(F.I.P.) if for every $\alpha \in(0,1]$, there exists $\epsilon, 0<\epsilon<\alpha$ such that for every finite subfamily $F_{1}, F_{2}, \ldots, F_{n}$ of $\mathcal{F}$, there exists $x \in X$ such that $\left(\bigcap_{i=1}^{n} F_{i}\right)(x)>1-\alpha+\epsilon$.

Theorem 4.1. Let $(X, \tau)$ be a fuzzy topological space. Then the following statements are equivalent:

(1) If $\mathcal{F}$ is a family of fuzzy closed sets satisfying finite intersection property (F.I.P.), then for each $\alpha \in(0,1]$, there exists $y \in X$ such that $\left(\bigcap_{F \in \mathcal{F}} F\right)(y)>1-\alpha$.

(2) $(X, \tau)$ is fuzzy compact. 
Proof. (1) $\Rightarrow(2)$ Let $\mathcal{G}$ be a family of fuzzy open sets in $X$ such that

$$
\begin{aligned}
& \alpha_{X} \subseteq \bigcup_{G_{i} \in \mathcal{G}} G_{i}, \alpha \in(0,1] \\
\Rightarrow & \bigcap_{G_{i} \in \mathcal{G}} G_{i}^{c} \subseteq(1-\alpha)_{X} \\
\Rightarrow & \nexists y \in X \text { such that }\left(\bigcap_{G_{i} \in \mathcal{G}} G_{i}^{c}\right)(y)>1-\alpha .
\end{aligned}
$$

Therefore in view of (1), $\mathcal{F}=\left\{G_{i}^{c}: G_{i} \in \mathcal{G}\right\}$ does not satisfy F.I.P., so for each $\epsilon$ such that $0<\epsilon<\alpha$, there exist $G_{1}^{c}, G_{2}^{c}, \ldots, G_{n}^{c} \in \mathcal{F}$ such that

$$
\begin{aligned}
& \left(\bigcap_{i=1}^{n} G_{i}^{c}\right)(x) \leq 1-\alpha+\epsilon, \quad \text { for each } x \in X \\
\Rightarrow & \bigcap_{i=1}^{n} G_{i}^{c} \subseteq(1-\alpha+\epsilon)_{X} \\
\Rightarrow & (\alpha-\epsilon)_{X} \subseteq \bigcup_{i=1}^{n} G_{i} \\
\Rightarrow & \alpha_{X} \text { is fuzzy compact. } \\
\Rightarrow & (X, \tau) \text { is fuzzy compact. }
\end{aligned}
$$

$(2) \Rightarrow(1)$ Conversely, assume that $(X, \tau)$ is fuzzy compact i.e., each $\alpha_{X}, \alpha \in[0,1]$, is fuzzy compact. Let $\mathcal{F}$ be a family of fuzzy closed sets satisfying F.I.P. We have to show that for each $\alpha \in(0,1]$, there exists $x \in X$ such that

$$
\left(\bigcap_{F \in \mathcal{F}} F\right)(x)>1-\alpha .
$$

Assume the contrary, i.e, for some $\alpha \in(0,1]$,

$$
\begin{aligned}
& \left(\bigcap_{F \in \mathcal{F}} F\right)(x) \leq 1-\alpha, \quad \forall x \in X \\
\Rightarrow & \bigcap_{F \in \mathcal{F}} F \subseteq(1-\alpha)_{X} \\
\Rightarrow & \alpha_{X} \subseteq \bigcup_{F \in \mathcal{F}} F^{c}
\end{aligned}
$$

This implies that $\left\{F^{c}: F \in \mathcal{F}\right\}$ is an open cover of $\alpha_{X}$. Since $\alpha_{X}$ is fuzzy compact, so for each $\epsilon$ such that $0<\epsilon<\alpha$, there exist $F_{1}^{c}, F_{2}^{c}, \ldots, F_{n}^{c} \in \mathcal{F}$ such that

$$
\begin{aligned}
& (\alpha-\epsilon)_{X} \subseteq \bigcup_{i=1}^{n} F_{i}^{c} \\
\Rightarrow & \bigcap_{i=1}^{n} F_{i} \subseteq(1-\alpha+\epsilon)_{X} \\
\Rightarrow & \nexists \text { any } x \text { such that }\left(\bigcap_{i=1}^{n} F_{i}\right)(x)>1-\alpha+\epsilon,
\end{aligned}
$$

implying that $\mathcal{F}$ does not satisfy F.I.P., which is a contradiction. 
Definition 4.2. [5] Let $\mathcal{R}$ be a fuzzy partial ordering on $X$ and $A \subseteq X$. Then the fuzzy upper bound for $A$ is the fuzzy set denoted by $U(\mathcal{R}, A)$ and defined by

$$
U(\mathcal{R}, A)=\bigcap_{x \in A} R_{x}, \text { where } R_{x}(y)=\mathcal{R}(x, y) \text {, for each } y \in X .
$$

Definition 4.3. A subset $A$ of $X$ is said to be $\alpha$-level bounded above, $\alpha \in(0,1]$, if for every $\epsilon, 0<\epsilon<\alpha$, there exists $y \in X$ such that

$$
U(\mathcal{R}, A)(y)>1-\alpha+\epsilon .
$$

We say that $A$ is fuzzy bounded above if it is $\alpha$-level bounded above, for each $\alpha \in(0,1]$.

Definition 4.4. A subset $A$ of $X$ is said to have $\alpha$-level least upper bound, $\alpha \in(0,1]$, if for every $\epsilon, 0<\epsilon<\alpha$, there exists $z \in X$ such that $U(\mathcal{R}, A)(z)>1-\alpha+\epsilon$ and $\mathcal{R}(z, y)>1-\alpha+\epsilon, \forall y$ such that $\mathcal{R}(x, y)>1-\alpha$, for each $x \in A$.

Definition 4.5. $A$ set $X$ is said to be $\alpha$-level complete if every $A \subseteq X$ which is $\alpha$-level bounded above has an $\alpha$-level least upper bound.

We say that $X$ is fuzzy complete if it is $\alpha$-level complete for every $\alpha \in(0,1]$.

Proposition 4.1. If $X$ is $\alpha$-level bounded above and $A \subseteq X$, then $A$ is also $\alpha$-level bounded above.

Proof. Since $X$ is $\alpha$-level bounded above, so for every $\epsilon, 0<\epsilon<\alpha$, there exists $y \in X$ such that

$$
U(\mathcal{R}, X)(y)>1-\alpha+\epsilon .
$$

We have to show that for every $\epsilon, 0<\epsilon<\alpha$, there exists $y \in X$ such that $U(\mathcal{R}, A)(y)>$ $1-\alpha+\epsilon$. Assume the contrary that for each $y \in X$,

$$
\begin{aligned}
& U(\mathcal{R}, A)(y) \leq 1-\alpha+\epsilon \\
\Rightarrow & \inf _{a \in A} \mathcal{R}(a, y) \leq 1-\alpha+\epsilon \\
\Rightarrow & U(\mathcal{R}, X)(y)=\inf _{x \in X} \mathcal{R}(x, y) \leq \inf _{a \in A} \mathcal{R}(a, y) \leq 1-\alpha+\epsilon, \text { for each } y \in Y
\end{aligned}
$$

which is a contradiction to the fact that $X$ is $\alpha$-level bounded above.

Theorem 4.2. Let $(X, \tau)$ be a fuzzy topological space. Then the following statements are equivalent:

(1) If $\mathcal{F}$ is a family of subbasic fuzzy closed sets satisfying finite intersection property (F.I.P.), then for each $\alpha \in(0,1]$, there exists $y \in X$ such that $\left(\bigcap_{F \in \mathcal{F}} F\right)(y)>1-\alpha$.

(2) For any subbase $\mathcal{S}$ of $\tau$, if $\mathcal{G} \subseteq \mathcal{S}$ such that $\alpha_{X} \subseteq \bigcup_{f \in \mathcal{G}} f$, then for each $\epsilon, 0<\epsilon<\alpha$, there exists a finite subset $\mathcal{G}_{0}$ of $\mathcal{G}$ such that $(\alpha-\epsilon)_{X} \subseteq \bigcup_{f \in \mathcal{G}_{0}} f$.

(3) $(X, \tau)$ is fuzzy compact.

Proof. We prove $(1) \Leftrightarrow(2)$. Let $\mathcal{S}$ be a subbase for $\tau$ and $\mathcal{G} \subseteq \mathcal{S}$ such that

$$
\begin{aligned}
& \alpha_{X} \subseteq \bigcup_{f \in \mathcal{G}} f \\
\Rightarrow & \bigcap_{f \in \mathcal{G}} f^{c} \subseteq(1-\alpha)_{X} \\
\Rightarrow & \nexists y \in X \text { such that }\left(\bigcap_{f \in \mathcal{G}} f^{c}\right)(y)>1-\alpha .
\end{aligned}
$$


Therefore in view of (1), $\mathcal{F}=\left\{f^{c}: f \in \mathcal{G}\right\}$ does not satisfy F.I.P., so for each $\epsilon$ such that $0<\epsilon<\alpha$, there exist $f_{1}^{c}, f_{2}^{c}, \ldots, f_{n}^{c} \in \mathcal{G}$ such that

$$
\begin{aligned}
& \left(\bigcap_{i=1}^{n} f_{i}^{c}\right)(x) \leq 1-\alpha+\epsilon, \quad \text { for each } x \in X \\
\Rightarrow & \bigcap_{i=1}^{n} f_{i}^{c} \subseteq(1-\alpha+\epsilon)_{X} \\
\Rightarrow & (\alpha-\epsilon)_{X} \subseteq \bigcup_{i=1}^{n} f_{i} .
\end{aligned}
$$

Conversely, assume that $\mathcal{F}$ be a family of subbasic fuzzy closed sets satisfying F.I.P. We have to show that for each $\alpha \in(0,1]$, there exists $x \in X$ such that

$$
\left(\bigcap_{F \in \mathcal{F}} F\right)(x)>1-\alpha .
$$

Assume the contrary i.e, for some $\alpha \in(0,1]$,

$$
\begin{aligned}
& \left(\bigcap_{F \in \mathcal{F}} F\right)(x) \leq 1-\alpha, \quad \forall x \in X \\
\Rightarrow & \bigcap_{F \in \mathcal{F}} F \subseteq(1-\alpha)_{X} \\
\Rightarrow & \alpha_{X} \subseteq \bigcup_{F \in \mathcal{F}} F^{c} .
\end{aligned}
$$

So according to our assumption, for each $\epsilon$ such that $0<\epsilon<\alpha$, there exist $F_{1}^{c}, F_{2}^{c}, \ldots, F_{n}^{c} \in$ $\mathcal{F}$ such that

$$
\begin{aligned}
& (\alpha-\epsilon)_{X} \subseteq \bigcup_{i=1}^{n} F_{i}^{c} \\
\Rightarrow & \bigcap_{i=1}^{n} F_{i} \subseteq(1-\alpha+\epsilon)_{X} \\
\Rightarrow & \nexists \text { any } x \text { such that }\left(\bigcap_{i=1}^{n} F_{i}\right)(x)>1-\alpha+\epsilon,
\end{aligned}
$$

implying that $\mathcal{F}$ does not satisfy F.I.P., which is a contradiction.

$(2) \Leftrightarrow(3)$ has already been proved in [7].

Theorem 4.3. Let $\mathcal{R}$ be a fuzzy partial order. If $X$ is fuzzy complete, fuzzy bounded above and $\mathcal{A}$ contains singletons, then $\left(X, \tau_{\mathcal{R}, \mathcal{A}}\right)$ is fuzzy compact.

Proof. Let $\mathcal{F}$ be a family of subbasic fuzzy closed sets of $X$ satisfying F.I.P. First, we show that there does not exist any $\delta_{X}$, where $\delta \in(0,1)$, belonging to $\mathcal{F}$. Since if we assume that $\mathcal{F}$ contains some $\delta_{X}$, where $\delta \in(0,1)$, then

$$
\bigcap_{F \in \mathcal{F}_{1}} F \subseteq \delta_{X}
$$


where $\mathcal{F}_{1}$ is a finite subfamily of $\mathcal{F}$ containing $\delta_{X}$, this implies that

$$
\bigcap_{F \in \mathcal{F}_{1}} F \subseteq\left(1-\alpha_{1}\right)_{X} \subseteq\left(1-\alpha_{1}+\epsilon\right)_{X}, \quad \text { for } \alpha_{1} \in(0,1) \text { such that } \delta=\left(1-\alpha_{1}\right) \text { and }
$$

for each $\epsilon, 0<\epsilon<\alpha_{1}$

$\Rightarrow \mathcal{F}$ does not satisfy F.I.P., which is a contradiction.

Next, if $\mathcal{F}$ contains only $1_{X}$, then the proof of the theorem is trivial and if there exists some member of the form $L_{\{x\}}$ or $R_{\{x\}}$ other than $1_{X}$ in $\mathcal{F}$, then $1_{X}$ plays no role in the intersection $\bigcap_{F \in \mathcal{F}} F$, so it is sufficient to prove the theorem for $\mathcal{F}$ of the form $\left\{L_{\left\{x_{\beta}\right\}}: \beta \in \Omega_{1}\right\} \cup\left\{R_{\left\{x_{\beta}\right\}}: \beta \in \Omega_{2}\right\}$. Now, we have to show that for each $\alpha \in(0,1]$, there exists $z \in X$ such that $\left(\bigcap_{F \in \mathcal{F}} F\right)(z)>1-\alpha$.

Let $A=\left\{x_{\beta}: \beta \in \Omega_{2}\right\} \subseteq X$. Since for $\alpha \in(0,1], X$ is $\alpha$-level bounded above, so by Proposition 4.1, $A$ is also $\alpha$-level bounded above. Therefore, by the fuzzy completeness of $X$, there exists an $\alpha$-level least upper bound of $A$. So, for each $\epsilon$ such that $0<\epsilon<\alpha$, there exists an element $x_{0} \in X$ such that

$$
\begin{aligned}
& U(\mathcal{R}, A)\left(x_{0}\right)>1-\alpha+\epsilon \text { and } \mathcal{R}\left(x_{0}, y\right)>1-\alpha+\epsilon, \forall y \in X \\
& \quad \text { such that } \mathcal{R}(x, y)>1-\alpha, \text { for each } x \in A \\
& \Rightarrow \inf _{x \in A} \mathcal{R}\left(x, x_{0}\right)>1-\alpha+\epsilon \\
& \Rightarrow \mathcal{R}\left(x_{\beta}, x_{0}\right)>1-\alpha+\epsilon, \quad \text { for each } \beta \in \Omega_{2} \\
& \Rightarrow R_{\left\{x_{\beta}\right\}}\left(x_{0}\right)>1-\alpha+\epsilon, \quad \text { for each } \beta \in \Omega_{2} .
\end{aligned}
$$

Since for $\beta \in \Omega_{2}, \mathcal{B}=\left\{R_{\left\{x_{\beta}\right\}}, L_{\left\{x_{\gamma}\right\}}\right\}$, where $\gamma \in \Omega_{1}$, is a finite subfamily of $\mathcal{F}$, so there exists $\epsilon_{1}, 0<\epsilon_{1}<\alpha$ and $y \in X$ such that

$$
\begin{aligned}
& \left(R_{\left\{x_{\beta}\right\}} \cap L_{\left\{x_{\gamma}\right\}}\right)(y)>1-\alpha+\epsilon_{1} \\
\Rightarrow & \min \left\{R_{\left\{x_{\beta}\right\}}(y), L_{\left\{x_{\gamma}\right\}}(y)\right\}>1-\alpha+\epsilon_{1} \\
\Rightarrow & \min \left\{\mathcal{R}\left(x_{\beta}, y\right), \mathcal{R}\left(y, x_{\gamma}\right)\right\}>1-\alpha+\epsilon_{1} \\
\Rightarrow & \left.\mathcal{R}\left(x_{\beta}, x_{\gamma}\right)>1-\alpha+\epsilon_{1}>1-\alpha \quad \text { (Using transitivity of } \mathcal{R}\right) .
\end{aligned}
$$

Since the above inequality holds for each $\beta \in \Omega_{2}$, so we have

$$
\begin{aligned}
& \left.\Rightarrow \mathcal{R}\left(x_{0}, x_{\gamma}\right)>1-\alpha+\epsilon \quad \text { (Using } 2 \text { and putting } y=x_{\gamma}\right) \\
& \Rightarrow L_{\left\{x_{\gamma}\right\}}\left(x_{0}\right)>1-\alpha+\epsilon .
\end{aligned}
$$

The above inequality holds for every $\gamma \in \Omega_{1}$. So

$$
L_{\left\{x_{\beta}\right\}}\left(x_{0}\right)>1-\alpha+\epsilon, \text { for each } \beta \in \Omega_{1} .
$$

From (3) and (4), we get

$$
\begin{aligned}
& \inf _{F \in \mathcal{F}} F\left(x_{0}\right) \geq 1-\alpha+\epsilon>1-\alpha \\
\Rightarrow & \left(\bigcap_{F \in \mathcal{F}} F\right)\left(x_{0}\right)>1-\alpha .
\end{aligned}
$$

Therefore by Theorem $4.2,\left(X, \tau_{\mathcal{R}, \mathcal{A}}\right)$ is fuzzy compact. 


\section{Conclusion}

In this paper, we have introduced fuzzy topologies genearted by fuzzy relations, as a generalization of the corresponding concept given by Smithson[11]. We have then obtained sufficient conditions under which this generated fuzzy topology satisfies seperation axioms, fuzzy $T_{0}$, fuzzy $T_{1}$ and fuzzy $T_{2}$. Further, we have introduced 'finite intersection property' in fuzzy topological spaces and obtained a characterization of Lowen's fuzzy compactness in terms of this property. Using this result, we have obtained a sufficient condition under which a fuzzy topology genearted by a fuzzy relation, becomes fuzzy compact.

\section{Acknowledgements}

The authors are very grateful to the editors and the referees for their valuable comments which led to the improvement of the paper.

\section{REFERENCES}

[1] Allam, A. A. , Bakeir, M. Y. and Tabl, E. A. Abo, (2008), Some methods for generating topologies by relations, Bull. Malays. Math. Sci. Soc.(2), 31, pp. 35-45.

[2] Campión, María Jesús, Candeal, Juan Carlos and Induráin, Esteban, (2009), Preorderable topologies and order-representability of topological spaces, Topology and its Applications, 156, pp. 2971-2978.

[3] Dalen, J. Van, and Wattle, E.,(1973), A topological characterization of ordered spaces, General Topology and its Applications, 3, pp. 347-354.

[4] Induráin, E. and Knoblauch, V., (2013), On topological spaces whose topology is induced by a binary relation, Quaestiones Mathematicae, 36, pp. 47-65.

[5] Klir, George J. and Yuan, Bo, (1997), Fuzzy Sets and Fuzzy Logic (Theory and Applications), Prentice Hall of India Private limited.

[6] Knoblauch, V., (2009), Topologies defined by binary relations, Department of Economics Working Paper Series, Working Paper 2009-28R, University of Connecticut.

[7] Lowen, R., (1976), Fuzzy topological spaces and fuzzy compactness, J. Math. Anal. Appl., 56, pp. 621-633.

[8] Lowen, R. and Srivastava, A. K., (1989), FTS $S_{0}$ : The epireflective hull of the Sierpinski object in FTS, Fuzzy Sets and Systems, 29, pp. 171-176.

[9] Ming, Pu Pao- and Ming, Liu Ying-,(1980), Fuzzy topology I. Neighborhood structure of a fuzzy point and Moore-Smith convergence, J. Math. Anal. Appl., 76, 571-599.

[10] Mishra, Seema and Srivastava, Rekha, (2018), Fuzzy topologies generated by fuzzy relations, Soft Computing, 22, pp. 373-385.

[11] Smithson, R. E., (1969), Topologies generated by relations, Bull. Austral. Math. Soc., 1, pp. 297-306.

[12] Srivastava, Rekha, Lal, S. N. and Srivastava, Arun K., (1981), Fuzzy Hausdorff Topological spaces, J. Math. Anal. Appl., 81, pp. 497-506.

[13] Srivastava, Rekha, Lal S. N. and Srivastava, Arun K., (1988), On Fuzzy $T_{1}$-Topological spaces, J. Math. Anal. Appl., 136, pp. 124-130.

[14] Zadeh, L. A., (1965), Fuzzy sets, Information and Control, 8, pp. 338-353.

[15] Zadeh, L. A., (1971), Similarity relations and fuzzy orderings, Inform. Sci. 3, pp. 177-200.

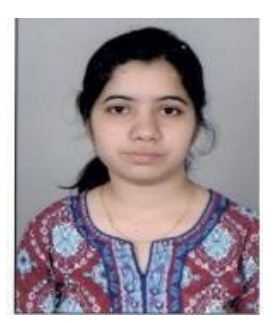

Seema Mishra has received her master's degree in 2012 from the University of Allahabad, India and Ph.D. in 2016 from Indian Institute of Technology (BHU), India. She is currently working as an assistant professor in the Department of Mathematics, Patna Women's College (Autonomous), Patna University, India. Her research interest is mainly in the fields of Fuzzy Topology, Fuzzy Soft Topology and Fuzzy Relations. She has published six research papers in these areas. 


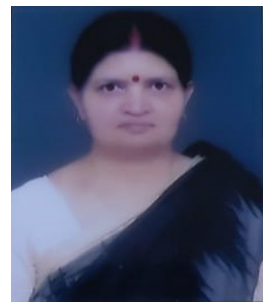

Rekha Srivastava earned her master's degree in 1976 from the University of Allahabad, India and Ph.D. in 1984 from Banaras Hindu University, India. Her research area is 'Fuzzy Topology', she has published several research papers in this area in reputed journals, including JMAA, FSS, Soft Computing, etc. and guided Ph.D. candidates in this area. Since 1984, she has been a member of the faculty in the Department of Applied Mathematics/Mathematical Sciences in the Indian Institute of Technology (BHU), Varanasi, India. She has been a professor in this department since 2008 